\title{
Virtual Negative Cable Resistance for Power Sharing Accuracy Enhancement in DC Microgrids
}

\author{
Fulong Li \\ Electrical, Electronic and Power \\ Engineering \\ Aston University \\ Birmingham, UK, B4 7ET \\ lif12@aston.ac.uk
}

\author{
Zhengyu Lin \\ Electrical, Electronic and Power \\ Engineering \\ Aston University \\ Birmingham, UK, B4 7ET \\ z.lin@ieee.org
}

\author{
Jiande $\mathrm{Wu}$ \\ College of Electrical \\ Engineering \\ Zhejiang University \\ Hangzhou, China, 310027 \\ eewjd@zju.edu.cn
}

\author{
Wuhua Li \\ College of Electrical \\ Engineering \\ Zhejiang University \\ Hangzhou, China, 310027 \\ woohualee@zju.edu.cn
}

\begin{abstract}
This paper proposed a secondary control method for power sharing accuracy enhancement and bus voltage restoration in DC microgrids. Droop control is notorious for its low power sharing accuracy when the cable resistance is considered. The virtual negative cable resistance (VNCR) concept is proposed compensate the cable resistance, therefore, it no longer has negative impact on the power sharing accuracy. The VNCR controller needs to monitor the DC bus voltage through low bandwidth communications. As the byproduct, the DC bus voltage restoration can be achieved simultaneously. The control signals are processed through low-pass filters, so the secondary control layer can be decoupled from the primary current/voltage controls over high frequency dynamics. Finally, the proposed VNCR method is validated through MATLAB simulation.
\end{abstract}

Keywords-bus voltage restoration, current sharing accuracy, DC microgrids, droop control, low pass filter.

\section{INTRODUCTION}

With the rapid penetration of renewable energy source (RES), such as wind power, solar power, hydro power, etc., the conventional power distribution structure is no longer costeffective. Microgrids [1] have been proposed for the power distribution of RESs with modern advanced power electronics technologies. DC microgrids, as one category of the microgrids, have been studied for their advantages [2] over the conventional AC microgrids, such as higher efficiency of energy utilization, less power transmission losses, no reactive power and harmonics, etc. A typical DC microgrid is shown in Fig. 1, and contains distributed source generations, energy storage devices, loads and controllable loads, such as electrical vehicles.

Usually there are many distributed power sources in a DC microgrid, and thus coordination of distributed sources is necessary for the effective operation of a DC microgrid. Based on this requirement, many control methods have been proposed in the last 10 years, such as master slave control [3], DC bus signalling [4][5], droop control [6-12], etc.

This work has received funding from the U.K. EPSRC UKRI Innovation Fellowship scheme under grant No. EP/S001662/1, and the European Union's Horizon 2020 research and innovation programme under grant agreement No. 734796 .

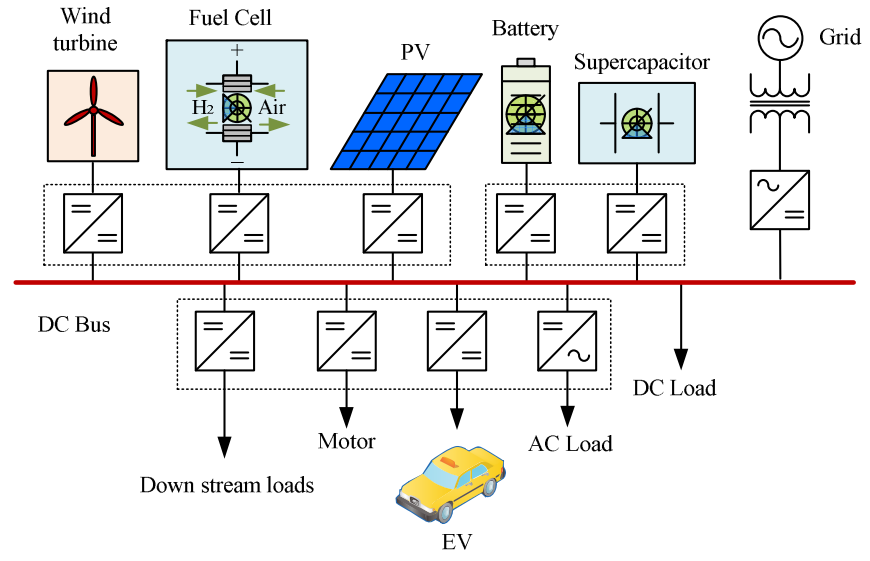

Fig. 1 A typical single DC bus microgrid configuration.

Droop control is one of the most applied control methods [2] in DC microgrids due to its easy implementation and distributed control structure. Droop control introduces virtual resistance for interface converters so that they are able to be parallel connected as voltage source. However, the main drawbacks of droop control are poor current sharing accuracy and significant DC bus voltage droop. These two drawbacks are both significantly affected by the line resistance. To put it simple, as the cable resistances increase, larger voltage deviations will be introduced and power sharing accuracy will be degraded.

In order to overcome the above drawbacks, a secondary control is needed to compensate the voltage deviations. The commonly applied scheme [2] is to monitor the DC bus voltage and add additional term in the voltage reference. This monitored DC bus voltage can then be transmitted through low bandwidth communications (LBC). There is a method [6] that does not use LBC but simply use a high-pass filter to block the DC component. However, it sacrifices the low frequency terminal impedance introduced by the droop control, and could also introduce large low frequency circulating current. In terms of current sharing accuracy, reference [7] uses the common current reference bus so that average current sharing can be achieved between the distributed energy sources. However, in real scenarios, flexible power sharing is usually required. Reference [8] uses voltage-shifting and slope-adjusting simultaneously to improve the current sharing accuracy, however, the cable 
resistances are not considered. Considering the cable resistance, the work in [9] uses LBC to exchange both current and voltage signals for DC bus voltage restorations and current sharing accuracy enhancement. However, when the load changes, the power sharing cannot be achieved properly, which has been pointed out in reference [10]. Besides, references [10] and [11] also consider the load changes when designing the controllers, and a Controller Area Network (CAN) bus communication interface is used. In addition, automatic droop coefficient is also proposed in [12] to adjust different loads.

By comparing and concluding the aforementioned DC bus voltage restoration and current sharing accuracy enhancement methods, it can be found that the existing literatures in DC microgrids are considering to adjust the virtual resistance or droop coefficient to improve the current sharing accuracy, and unavoidably, it can always be affected by the cable resistance. This paper proposes an intact control method for the secondary control layer for eliminating the impact of cable resistance on power sharing accuracy and DC bus voltage restorations. Firstly, the concept of virtual negative cable resistance (VNCR) is proposed and used together with conventional virtual resistance in droop control. The virtual negative cable resistance can be used to compensate the real cable resistance so the power sharing between the distributed sources only determined by the original designed virtual resistance. In this sense, only the DC bus voltage signal is required for the loop feedback, which can be achieved through LBC. Meanwhile, the DC bus voltage is also used for determining the voltage deviations so that the $\mathrm{DC}$ bus voltage restoration can be achieved by adding this additional term as conventional ways.

The rest of this paper is organized as follows: Section II briefly illustrates the problems introduced by droop control in DC microgrids. Section III introduces the principles of proposed methods and the detailed control design is illustrated. Section IV shows the simulation results. Finally, conclusions are given in Section V.

\section{ANALYSIS OF DROOP CONTROL}

\section{A. Brief Introduction of Droop Control}

When two tightly regulated interface converters are parallel connected, it will generate the large circulating current if there exist minor terminal voltage variations, which is shown in Fig. 2. Introducing the virtual resistance reduces the circulating current and it can be used to the regulate the power sharing between the distributed sources as the voltage sources.

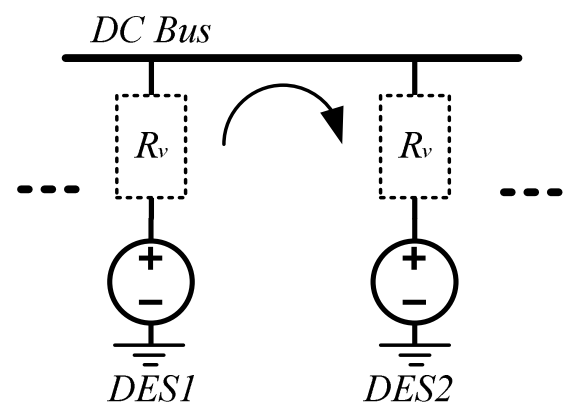

Fig. 2 Equivalent model of two parallel connected distributed energy sources.
Based on the conventional droop control, as is shown above, the current and voltage relationship of distributed sources can be written in equation (1).

$$
v_{k}^{\prime}=v_{k}^{*}-i_{o k} R_{v k}, k \in\{1,2, \cdots, n\}
$$

where $v_{k}^{\prime}$ is the control loop voltage reference; $v_{k}^{*}$ is the floating voltage or nominal voltage; $R_{v k}$ is the virtual resistance (droop coefficient), $i_{o k}$ is terminal output current; $n$ is total number of converters connected in the system; $k$ is the $k$-th converter.

Droop control is applied in the above conventional voltage/ current control loop. It introduces the virtual resistance to vary the voltage reference, so the terminal voltage drops when the load increases, which is shown in Fig. 3.
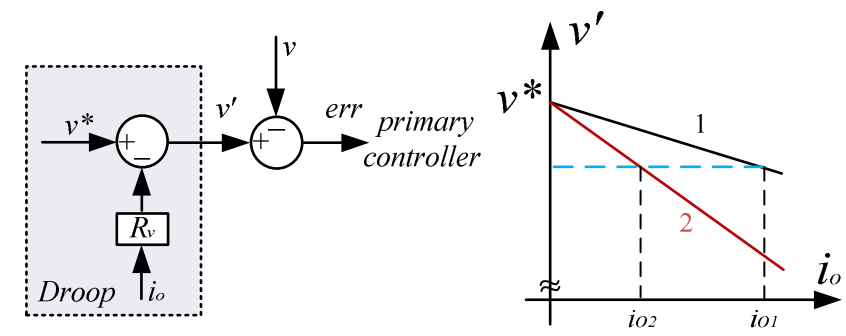

Fig. 3 Control blocks of conventional droop control and droop curves.

In an ideal case, neglecting the cable resistance, the power/current sharing between the distributed energy sources is simply determined by the ratio of droop coefficient.

\section{B. Problem Statements}

When considering the cable resistance, the power sharing between the DESs is no longer virtual resistance dependent only. It can be significantly impacted by the cable resistance. The diagram considering the cable resistance is shown in Fig. 4.

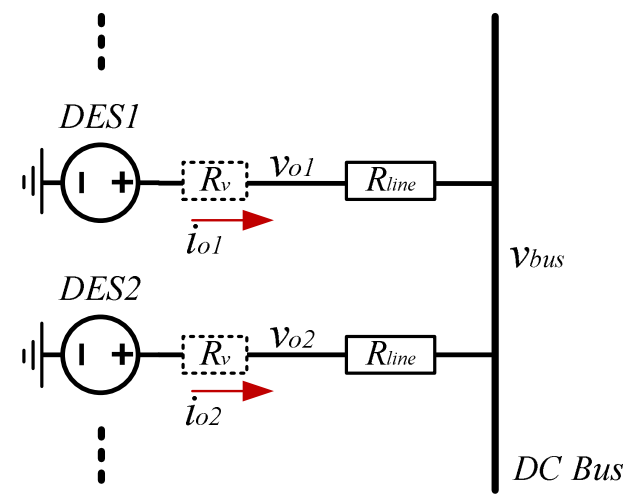

Fig. 4 Equivalent model considering cable resistance.

The total terminal resistance is written as equation (2).

$$
R_{t}=R_{v}+R_{\text {line }}
$$

If assuming the floating voltages $v^{*}$ are designed same on the interface converters, when they are parallel connected with same DC bus, then equation (3) can be attained. 


$$
v^{*}-i_{01} R_{t 1}=v^{*}-i_{02} R_{t 2}
$$

By solving above equations, the current sharing in the two distributed energy sources can be written as equation (4).

$$
\rho=\frac{i_{01}}{i_{02}}=\frac{R_{t 2}}{R_{t 1}}=\frac{R_{v 2}+R_{\text {line } 2}}{R_{v 1}+R_{\text {line } 1}}
$$

where $\rho$ is power sharing ratio.

Generally, if all the distributed energy sources are symmetrically connected on the DC bus, the current sharing can be simply tracked from either virtual resistance or cable resistance. This means when $R_{v} \gg R_{\text {line }}$, the current sharing will be determined by the virtual resistance, which means $\rho \rightarrow$ $R_{v 2} / R_{v 1}$. This is corresponded to the engineer's designed purpose; when $R_{v} \ll R_{\text {line }}$, the cable resistance will be responsible for the power/sharing, which means $\rho \rightarrow$ $R_{\text {line } 2} / R_{\text {line } 1}$. In real applications, the cable resistance can be affected by the environment, such as temperatures. This will the make the power sharing uncontrollable.

One of the effective methods is to increase the virtual resistance, thus make it larger than the cable resistance, the power sharing between the DESs will be dominated by the virtual resistance. Therefore, the cable resistance has less impact on power sharing accuracy. However, increasing the virtual resistance, the voltage droop will increase as well. Besides, it will also introduce the concerns on the stability performance [9]. Even though the voltage droop can be compensated through the LBC based methods, the impact of large droop coefficient on effectiveness of primary controller cannot be overlooked. Admittedly, it is also worthwhile to discuss the stability performance while altering the controllers. However, only the controllers design is introduced here in order to make this paper focused.

\section{EXPLANATIONS OF PROPOSED SECONDARY CONTROLS}

\section{A. Proposed Negative Cable Resistance Compensations}

The cable resistance impacts the power sharing accuracy as aforementioned. Inspired by the virtual resistance, it is possible to produce a negative virtual cable resistance to compensate the line resistance, as shown in Fig. 5.

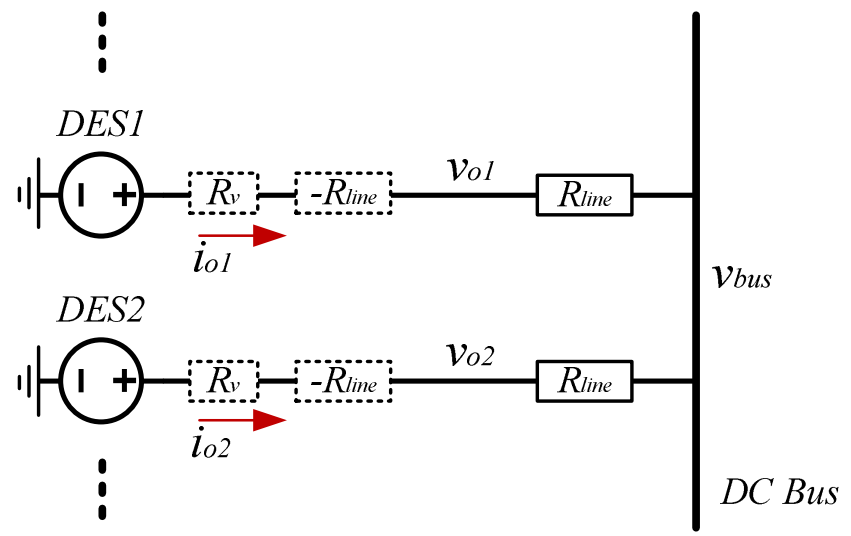

Fig. 5 Negative resistance introduced in previous model.
Based on this idea, the next thing is to consider how to introduce the proposed negative cable resistance concept in the control blocks. The cable resistance can be written as equation (5).

$$
R_{\text {line }}=\frac{v_{o k}-v_{b u s}}{i_{o k}}
$$

The virtual resistance can be written as equation (6).

$$
R_{v}=\frac{v_{k}^{*}-v_{k}^{\prime}}{i_{o k}}
$$

Therefore, the superposing equation (5) and equation (6) in equation (2), the total introduce virtual resistance is shown in equation (7).

$$
\begin{aligned}
R_{t k}=\frac{v_{o k}-v_{b u s}}{i_{o k}} & +\frac{v_{k}^{*}-v^{\prime}}{i_{o k}} \\
= & \frac{v_{k}^{*}+v_{o k}-v_{b u s}-v_{k}^{\prime}}{i_{o k}}
\end{aligned}
$$

By drawing above equation in the control blocks, the secondary virtual negative cable resistance can be shown in Fig. 6.

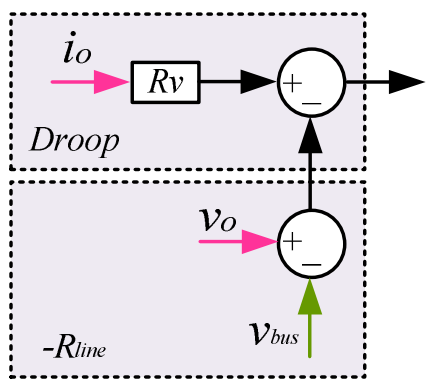

Fig. 6 Virtual negative cable resistance compensation in control blocks.

It can be seen that apart from the parameters $\left(i_{o}\right.$ and $\left.v_{o}\right)$ from the primary control loops, the $v_{\text {bus }}$ need to be attained over the cable through the LBC. Then cable resistance can be eliminated by the proposed negative resistance, therefore, the current sharing accuracy will no longer be impacted by the cable resistance.

\section{B. DC Bus Voltage Restorations}

Coincidentally, the signal $v_{\text {bus }}$ from LBC can also be used for the DC bus voltage restoration from previous literatures. DC bus voltage droop is caused by the virtual resistance and cable resistance. The easiest way is to add the voltage droop term $\delta$ to compensate the voltage droop, therefore, the terminal voltage of interface converter will adjust based on the voltage droop to keep the bus voltage constant all the time. This process can be written as equation (8).

$$
v_{k}^{\prime}=v_{k}^{*}-i_{o k} R_{t k}+\delta
$$

Similarly, applying above equation on the controls, the control blocks can be drawn in Fig. 7 . 


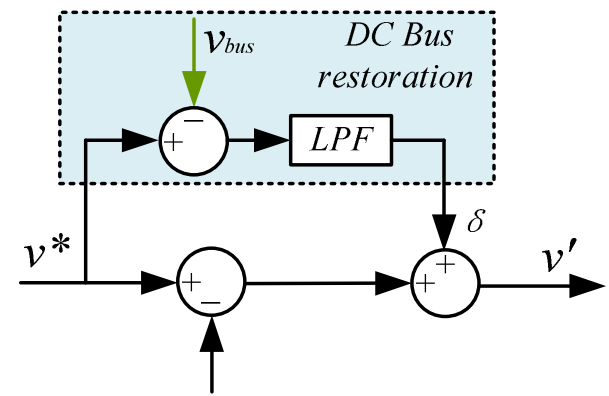

Fig. 7 Control blocks of bus voltage restorations.

One thing worth to mention is that the external control signal $v_{\text {bus }}$ is transmitted through LBC, it can be configured as distributed way or centralized way. Considering centralized control scheme, a central monitor is required for detecting the DC bus voltage. However, the centralized configuration is notorious for its single point of failure. Therefore, the DC bus voltage needs to be monitored in each converters' terminals if implementing the system in a distributed way. In this paper, a distributed way is chosen to line up with the distributed characteristics of droop control.

\section{CONTROLLERS DESIGN}

The proposed secondary controllers is based on a bidirectional buck-boost converter, which is shown in Fig. 8.

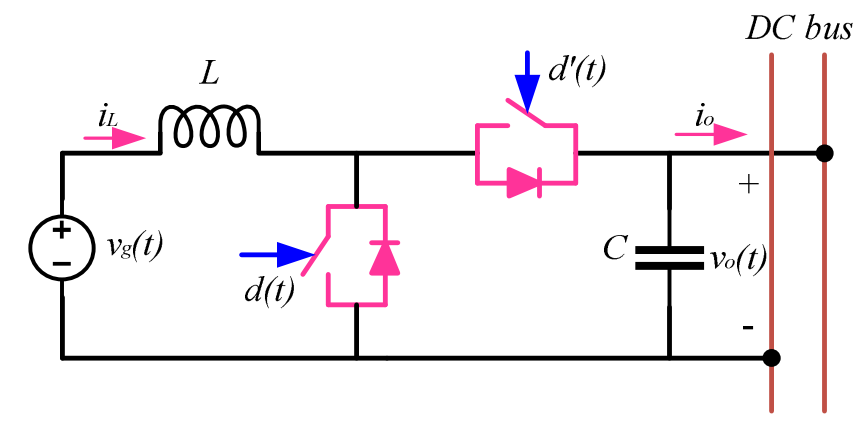

Fig. 8 The bidirectional converter in this research.

The system dynamic equations are listed below.

$$
\left\{\begin{array}{l}
L \frac{d i_{L}(t)}{d t}=v_{g}(t)-d^{\prime}(t) v_{o}(t) \\
C \frac{d v_{o}(t)}{d t}=d^{\prime}(t) i_{L}(t)-i_{o}(t)
\end{array}\right.
$$

where $v_{g}$ is input source voltage; $v_{o}$ is converter output voltage; $d$ is the duty cycle and $d^{\prime}$ equals to $1-d ; i_{o}$ is output current and $i_{L}$ is inductor current.

Applying small signal analysis based equations, the converter transfer functions of control to inductors and output voltage can be easily attained. By combining the control blocks designed in the last section, the intact controller as shown in Fig. 9 is designed. It can be seen that only one signal comes from the $\mathrm{LBC}$, and the rest control signals comes from the previous control loops, such as $i_{o}$ and $v_{o}$, which simplify the system complicity and increase the system reliability.

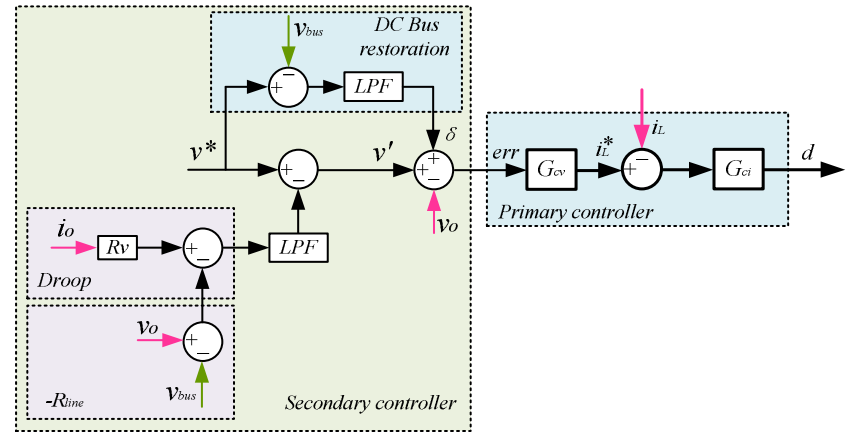

Fig. 9 Intact secondary control with enhanced current sharing accuracy and bus restoration.

Based on Fig. 9, the controller designs can also be divided into two parts, which are primary controller and secondary controller, respectively.

\section{A. Primary controllers}

The inner controller loop can be designed from conventional bode plot methods by ensuring sufficient margins based on the transfer functions. The structures of current and voltage controllers are designed as follows.

$$
\begin{gathered}
G_{c i}=G_{i m} \cdot \frac{1+\frac{\omega_{z}}{S}}{1+\frac{s}{\omega_{p}}} \\
G_{c v}=G_{v m} \cdot\left(1+\frac{\omega_{z v}}{s}\right)
\end{gathered}
$$

The inner closed loop and outer closed loop margins is shown in Fig. 10. The data for the margin test are listed in Table I. It can be seen that the proposed primary controllers can ensure the stable operation of this system and have sufficient margins.

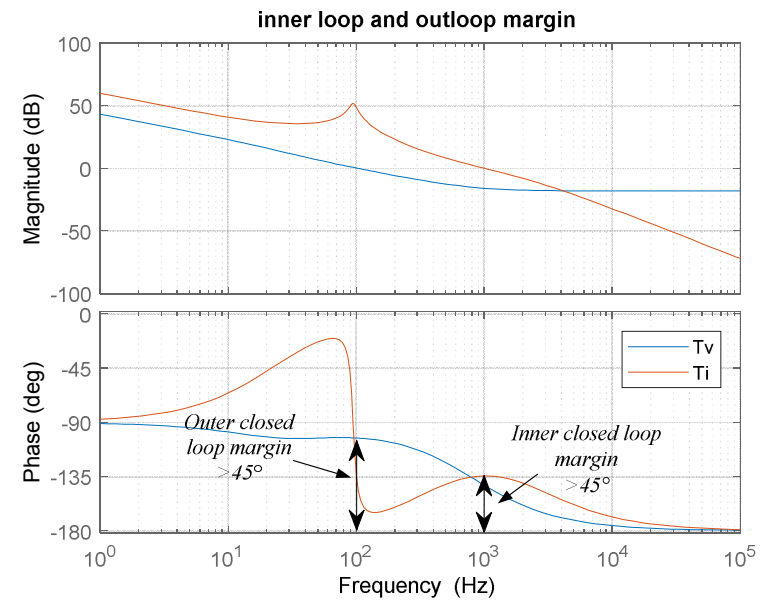

Fig. 10 Inner loop and outer loop margin test.

\section{B. Secondary controllers}

The introduced NVCR and DC bus restoration terms are both required with DC component ideally. In the control blocks, it can be seen the sampled the voltage and current contains the 
whole frequency dynamics, it is thus necessary to add a low pass filter to suppress the high frequency interactions. The low pass filters are designed as follows.

$$
\begin{gathered}
G_{N V C R}^{L P F}=\frac{1}{1+\frac{s}{2 \pi f_{c 1}}} \\
G_{B V R}^{L P F}=\frac{1}{1+\frac{s}{2 \pi f_{c 2}}}
\end{gathered}
$$

where $f_{c 1}$ is the crossover frequency of negative virtual cable resistance control; $f_{c 2}$ is the crossover frequency of bus voltage restoration control.

The NVCR control is tend to be faster than the bus voltage restorations, which means that $f_{c 1}>f_{c 2}$ and the bandwidth of total secondary control is smaller than the primary control from design considerations.

\section{Simulation Results}

Based on the above analysis, the proposed method for enhancing the power sharing accuracy and bus restoration are validated through Matlab/Simulink simulation. The system configuration is shown in Fig. 11.

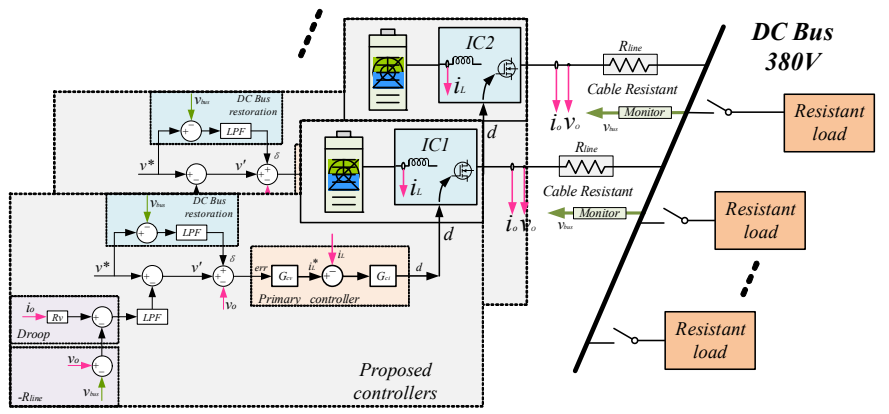

Fig. 11 System configuration in the simulation.

The parameters used in the simulation are listed in Table I. The simulation contains the three parts. The first one is DC bus voltage restorations; the second one is NVCR compensations; and the last one is combining cable resistance and load disturbation test.

TABLE I. PARAMETERS AND VALUES OF SIMULATIONS.

\begin{tabular}{|c|c|c|c|}
\hline Parameters & Values & Parameters & Values \\
\hline$L / C$ & $1.2 \mathrm{mH} / 470 u \mathrm{~F}$ & $f_{z} / \omega_{z}$ & $400 \mathrm{~Hz}$ \\
\hline$f_{s}$ & $10 \mathrm{kHz}$ & $f_{p} / \omega_{p}$ & $2.5 \mathrm{kHz}$ \\
\hline$R_{v}$ & $1 \sim 10 \Omega$ & $f_{z v} / \omega_{z v}$ & $33.3 \mathrm{~Hz}$ \\
\hline$R_{\text {line }}$ & $1 \sim 10 \Omega$ & $f_{c 1}$ & $80 \mathrm{~Hz}$ \\
\hline $\begin{array}{c}\text { Input } \\
\text { sources }\end{array}$ & $150 \sim 170 \mathrm{~V}$ & $f_{c 2}$ & $1 \sim 10 \mathrm{~Hz}$ \\
\hline Load & $0 \sim 5 \mathrm{~kW}$ & $v_{\text {bus }}$ & $380 \mathrm{~V}$ \\
\hline \multicolumn{4}{|r}{} \\
\end{tabular}

\section{A. DC bus restorations}

The simulation results of DC bus restoration is shown in Fig. 12. In this case, the droop coefficient is chosen as $10 \Omega$ for showing the effectiveness of proposed secondary controller. It can be seen that large voltage droop is stimulated, which is nearly $20 \mathrm{~V}$. The controller of DC bus restoration is switched on at $0.1 \mathrm{~s}$. It can be seen that bus voltage is restored to $380 \mathrm{~V}$ as expected.
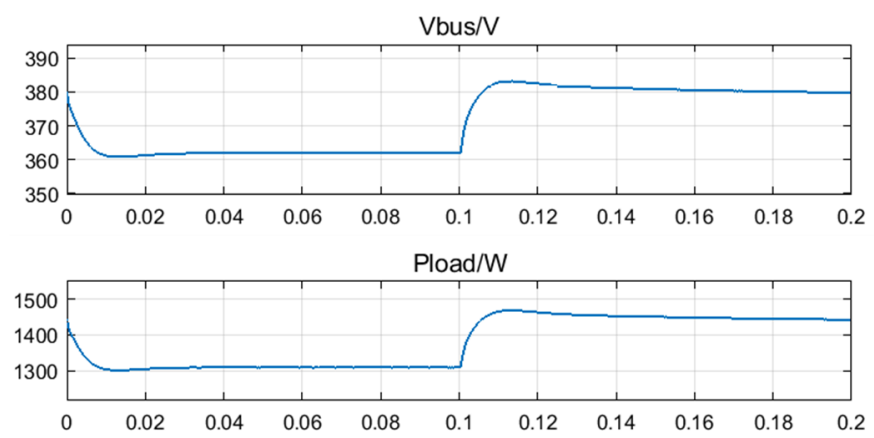

Offset=0

Fig. 12 Validation of DC bus voltage restorations.

\section{B. NVCR compensations}

The simulation result of NVCR compensation is shown in Fig. 13. The droop coefficients in this case are $1 \Omega$ for both converters. Theoretically, the power sharing should be equal. However, impacted by the cable resistance $(1 \Omega$ cable resistance for converter 1 and $2 \Omega$ cable resistance for converter 2 ), it can be seen that the power sharing between the two sources are not equal. Therefore, the current sharing accuracy is deteriorated. After NVCR controller is switched on at $0.1 \mathrm{~s}$, the power sharing is adjusted to equality automatically. Due to the DC bus voltage restoration is also implemented, the bus voltage is kept at $380 \mathrm{~V}$.
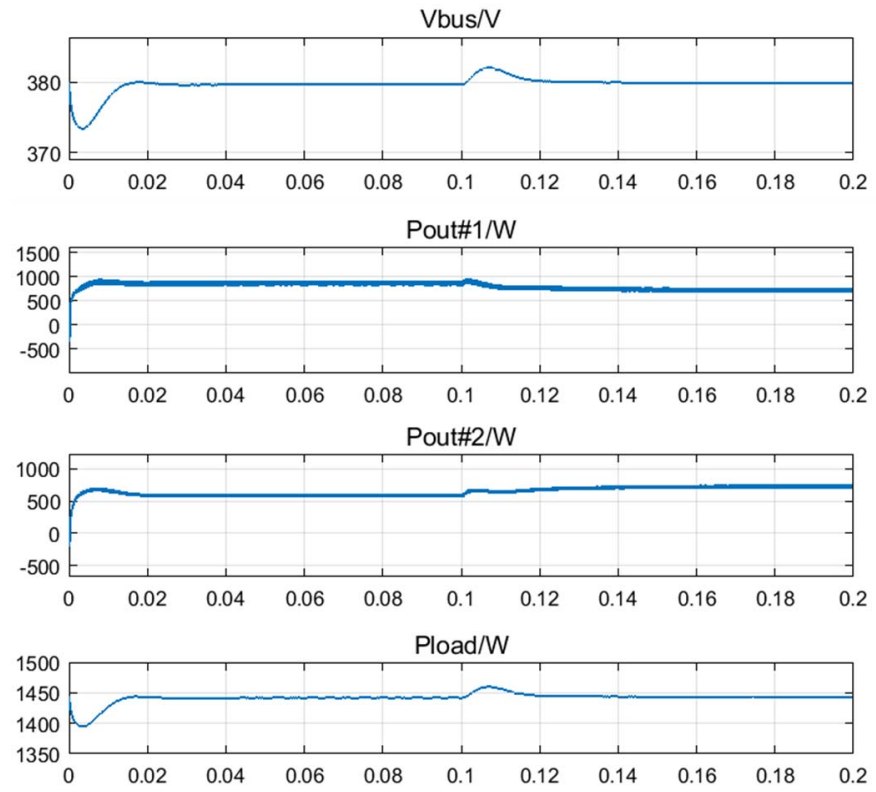

Fig. 13 Validation of NVCR compensation. 


\section{Disturbation test}

The last case shows that the engineers or upper layer controllers can regulate the power by simply tuning virtual resistance, and no longer affected by the cable resistance. The droop coefficient in converter 1 is set as $1 \Omega$, and $2 \Omega$ in converter 2 . Theoretically, the power output in converter 1 is two times of converter 2. The results shown in Fig. 14 is same as predicted power sharing principle. the cable resistance is disturbed at $0.05 \mathrm{~s}$, yet the power sharing and bus voltage restoration can be controlled well. Besides, when the load step from $1.44 \mathrm{~kW}$ to $2.89 \mathrm{~kW}$ at $0.1 \mathrm{~s}$, the proportional power sharing can still be same with designed power sharing ratio.
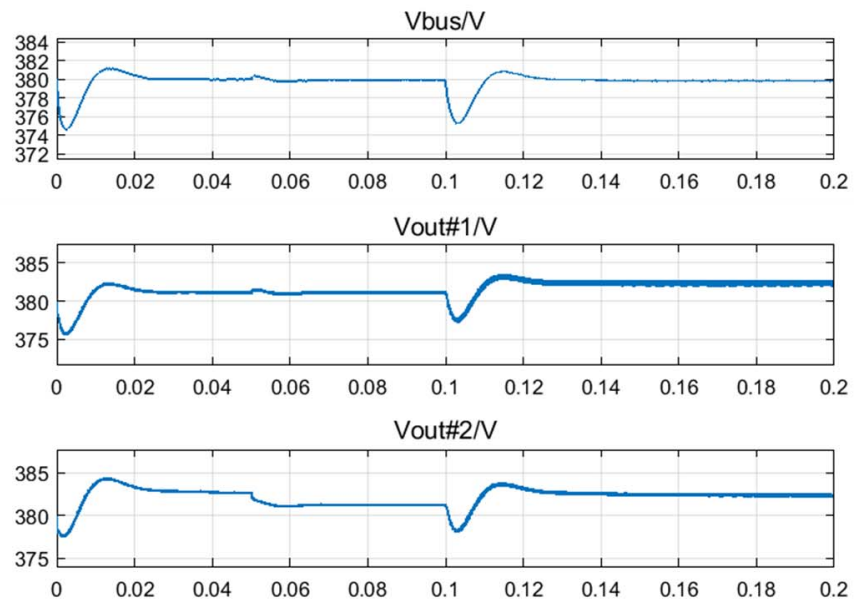

Pout\#1/W

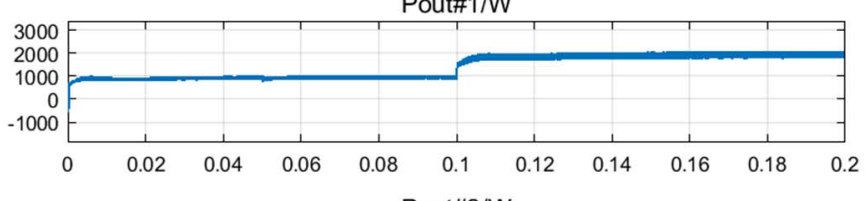

Pout\#2/W
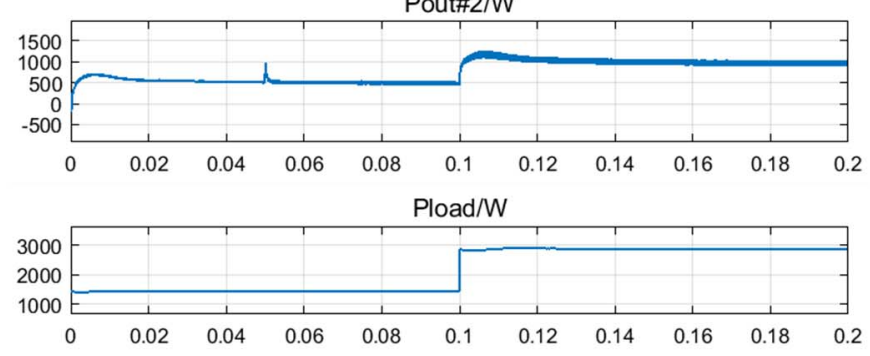

Fig. 14 Mixed dynamic test for NVCR and DC bus restorations.

\section{CONCLUSIONS}

This paper proposed a method that utilizes the DC bus voltage information transmitted through low band-width communications to achieve the both DC bus restorations and accurate power sharing. A concept of virtual negative cable resistance is proposed and used for compensate the impact from cable resistance on the power sharing such that the cable resistances are no more affect the power sharing accuracy. The design of the proposed secondary controller is illustrated, and MATLAB simulation results validate the proposed method.

\section{REFERENCES}

[1] R. H. Lasseter, "MicroGrids," in 2002 IEEE Power Engineering Society Winter Meeting. Conference Proceedings (Cat. No.02CH37309), vol. 1, pp. 305-308.

[2] J. M. Guerrero, J. C. Vasquez, J. Matas, L. G. De Vicuña, and M. Castilla, "Hierarchical control of droop-controlled AC and DC microgrids - A general approach toward standardization," IEEE Trans. Ind. Electron., vol. 58, no. 1, pp. 158-172, Nov. 2011.

[3] J. Rajagopalan, K. Xing, Y. Guo, F. C. Lee, and B. Manners, "Modeling and dynamic analysis of paralleled DC/DC converters with master-slave current sharing control," Proc. Appl. Power Electron. Conf. APEC '96, vol. 2, pp. 678-684, 1996.

[4] J. Schonbergerschonberger, R. Duke, and S. D. Round, "DC-Bus Signaling: A Distributed Control Strategy for a Hybrid Renewable Nanogrid," IEEE Trans. Ind. Electron., vol. 53, no. 5, pp. 1453-1460, Oct. 2006.

[5] F. Li, Z. Lin, Z. Qian, and J. Wu, "Active DC bus signaling control method for coordinating multiple energy storage devices in DC microgrid," in 2017 IEEE Second International Conference on DC Microgrids (ICDCM), 2017, pp. 221-226.

[6] Q. Xu, J. Xiao, X. Hu, P. Wang, and M. Y. Lee, “A Decentralized Power Management Strategy for Hybrid Energy Storage System With Autonomous Bus Voltage Restoration and State-of-Charge Recovery," IEEE Trans. Ind. Electron., vol. 64, no. 9, pp. 7098-7108, Sep. 2017.

[7] S. Anand, B. G. Fernandes, and J. M. Guerrero, "Distributed control to ensure proportional load sharing and improve voltage regulation in lowvoltage DC microgrids," IEEE Trans. Power Electron., vol. 28, no. 4, pp. 1900-1913, Apr. 2013.

[8] P. Wang, X. Lu, X. Yang, W. Wang, and D. Xu, "An Improved Distributed Secondary Control Method for DC Microgrids with Enhanced Dynamic Current Sharing Performance," IEEE Trans. Power Electron., vol. 31, no. 9, pp. 6658-6673, Sep. 2016.

[9] X. Lu, J. M. Guerrero, K. Sun, and J. C. Vasquez, "An improved droop control method for dc microgrids based on low bandwidth communication with dc bus voltage restoration and enhanced current sharing accuracy," IEEE Trans. Power Electron., vol. 29, no. 4, pp. 1800-1812, Apr. 2014.

[10] D.-H. Dam and H.-H. Lee, "A Power Distributed Control Method for Proportional Load Power Sharing and Bus Voltage Restoration in a DC Microgrid," IEEE Trans. Ind. Appl., vol. 54, no. 4, pp. 3616-3625, Jul. 2018.

[11] P. H. Huang, P. C. Liu, W. Xiao, and M. S. El Moursi, "A Novel DroopBased Average Voltage Sharing Control Strategy for DC Microgrids," IEEE Trans. Smart Grid, vol. 6, no. 3, pp. 1096-1106, May 2015.

[12] R. Iravani, A. Khorsandi, M. Ashourloo, and H. Mokhtari, "Automatic droop control for a low voltage DC microgrid," IET Gener. Transm. Distrib., vol. 10, no. 1, pp. 41-47, Jan. 2016. 